\section{OP0224 SUSTAINED REDUCTIONS IN INFLAMMATORY BIOMARKERS IN PATIENTS WITH MODERATE TO SEVERE PSORIATIC ARTHRITIS}

D. D. Gladman ${ }^{1}$, W. Jiang ${ }^{2}$, A. Hertz², V. Malkov², O. K. Yoon ${ }^{2}$, M. Trivedi' ${ }^{2}$ M. Alani ${ }^{2,3}$, L. Vestergaard' ${ }^{2}$, R. Besuyen ${ }^{4}$, R. Galien ${ }^{5}$, A. M. Mirza ${ }^{2}$, V. Chandran ${ }^{1}{ }^{1}$ University of Toronto and Krembil Research Institute, Toronto Western Hospital, Toronto, Canada; ' ${ }^{2}$ Gilead Sciences Inc., Foster City, United States of America; ${ }^{3}$ University of Washington, Seattle, United States of America; ${ }^{4}$ Galapagos BV, Leiden, Netherlands; ${ }^{5}$ Galapagos SASU, Romainville, France

Background: Psoriatic arthritis (PsA) is a chronic inflammatory musculoskeletal disease characterized by musculoskeletal and cutaneous inflammation. In the recent EQUATOR study (NCT03101670), patients (pts) with active PsA receiving the oral, selective Janus kinase 1 (JAK1) inhibitor filgotinib (FIL) had significant and sustained improvements versus placebo (PBO) in clinical signs and symptoms. We present here updated results of the EULAR 2019 presentation of EQUATOR on circulating biomarkers in PSA.

Objectives: To evaluate the impact of FIL on the levels of circulating proinflammatory cytokines and chemokines, adhesion molecules, and markers of matrix remodeling in EQUATOR pts with active PsA

Methods: EQUATOR was a 16-week, double-blind, multicenter, Phase 2 study in pts with active PsA. Pts were randomized 1:1 to FIL $200 \mathrm{mg}(\mathrm{n}=65)$ or PBO ( $n=66)$ once daily. Serum samples (FIL $n=60$ and PBO $n=61$ ) were collected at baseline (BL) and at Weeks 1, 4, and 16. The association of BL biomarkers with PsA disease characteristics was analyzed by Spearman's rank-order correlation. Biomarker changes from BL were assessed in time-paired serum samples using multiplex and high sensitivity ELISA-based assays. Analytes were grouped by hierarchical clustering; treatment effect on a biomarker was defined as a difference in change from BL between pts receiving FIL versus PBO. Improvements in PsA clinical signs and symptoms were determined by assessing changes from $\mathrm{BL}$ in a number of clinical disease activity scores including psoriatic arthritis disease activity score (PASDAS), psoriasis area and severity index (PASI) and disease activity index for psoriatic arthritis (DAPSA) scores.

Results: BL levels of numerous biomarkers were associated $(p<0.05)$ with clinical measures of PsA. Several clusters of biomarkers were identified based on the rate and magnitude of FIL treatment response. Cluster 1 included biomarkers with substantial reductions from BL with FIL by week 1 , such as the acute phase proteins CRP and SAA (>50\%), and the inflammatory mediators IL-6, CXCL10, and IL-23 (>25\%). Cluster 2 included biomarkers of cell adhesion (ICAM-1, VCAM1) with a $5 \%-15 \%$ reduction from BL with FIL by week 1 . Cluster 3 included biomarkers of matrix remodeling (MMP1, SC1M) with a delayed $>25 \%$ reduction from BL with FIL that was significant by Week 4. Finally, Cluster 4 included biomarkers with a modest $(5 \%-10 \%)$ increase from BL with FIL (Eotaxin, IL-15, and adiponectin). Spearman rank correlation analyses showed that at BL, many biomarkers were positively associated with disease scores, and tended to segregate between psoriasis weighted scores such as PASI and arthritis weighted scores such as DAPSA. The observed decrease in proinflammatory cytokines were associated with on-treatment improvements from BL in disease score for pts receiving FIL. Conclusion: Compared with PBO, FIL significantly decreased BL levels of circulating biomarkers associated with PsA disease activity, including proinflammatory cytokines and chemokines, adhesion molecules, and markers of matrix remodeling. The observed decreases in circulating proinflammatory cytokines and biomarkers of both bone pathobiology and psoriatic disease suggest that FIL improves PsA clinical signs and symptoms at a molecular level. These findings are consistent with reduced disease activity in pts with PsA and suggest that FIL treatment leads to a rapid and sustained reduction of inflammation in PsA.

Acknowledgments: This study was funded by Gilead Sciences, Inc. Editorial support was provided by Fishawack Communications Inc and funded by Gilead Sciences, Inc.

Disclosure of Interests: Dafna D Gladman Grant/research support from: AbbVie, Amgen Inc., BMS, Celgene Corporation, Janssen, Novartis, Pfizer, UCB - grant/research support, Consultant of: AbbVie, Amgen Inc., BMS, Celgene Corporation, Janssen, Novartis, Pfizer, UCB - consultant, Wendy Jiang Shareholder of: Gilead Sciences Inc., Employee of: Gilead Sciences Inc., Angie Hertz Shareholder of: Gilead Sciences Inc, Employee of: Gilead Sciences Inc, Vlad Malkov Shareholder of: Gilead Sciences Inc., Employee of: Gilead Sciences Inc., Oh Kyu Yoon Shareholder of: Gilead Sciences Inc., Employee of: Gilead Sciences Inc., Mona Trivedi Shareholder of: Amgen and Gilead Sciences, Employee of: Gilead Sciences, Muhsen Alani Employee of: Gilead Sciences, Lene Vestergaard Shareholder of: Gilead Sciences Inc., Employee of: Gilead Sciences Inc., Robin Besuyen Shareholder of: Galapagos, Employee of: Galapagos, René Galien Shareholder of: Galapagos, Employee of: Galapagos, Amer M. Mirza Shareholder of: Gilead Sciences Inc., Employee of: Gilead Sciences
Inc., Vinod Chandran Grant/research support from: Abbvie, Celgene, Consultant of: Abbvie, Amgen, Bristol-Myers Squibb, Celgene, Eli Lily, Janssen, Novartis, Pfizer, UCB, Employee of: Spouse employed by Eli Lily DOI: 10.1136/annrheumdis-2020-eular.4927

\section{\begin{tabular}{|l|l}
\hline OP0225 TOFACITINIB AS MONOTHERAPY FOLLOWING \\
\hline
\end{tabular} METHOTREXATE WITHDRAWAL IN PATIENTS WITH PSORIATIC ARTHRITIS PREVIOUSLY TREATED WITH OPEN-LABEL TOFACITINIB + METHOTREXATE: A RANDOMISED, PLACEBO-CONTROLLED SUBSTUDY OF OPAL BALANCE}

P. Nash ${ }^{1}$, L. C. Coates ${ }^{2}$, P. J. Mease ${ }^{3}$, A. Kivitz ${ }^{4}$, D. D. Gladman ${ }^{5}$, F. Behrens ${ }^{6}$, J. C. C. Wei ${ }^{7}$, D. Fleishaker ${ }^{8}$, J. Wu' ${ }^{9}$, C. Wang ${ }^{8}$, A. B. Romero ${ }^{10}$, L. Fallon ${ }^{11}$ M. A. Hsu ${ }^{8}$, K. Kanik ${ }^{8} .{ }^{1}$ Griffith University, Brisbane, Australia; ${ }^{2}$ University of Oxford, Oxford, United Kingdom; ${ }^{3}$ Swedish Medical Center and University of Washington, Seattle, United States of America; ${ }^{4}$ Altoona Center for Clinical Research, Duncansville, United States of America; ${ }^{5}$ University of Toronto, Toronto, Canada; ${ }^{6}$ Goethe University, Frankfurt, Germany; ${ }^{7}$ Chung Shan Medical University Hospital, Taichung, Taiwan, Republic of China; ${ }^{8}$ Pfizer Inc, Groton, United States of America; ${ }^{8}$ Pfizer Inc, Groton, United States of America;

${ }^{10}$ Pfizer Inc, Barcelona, Spain; ${ }^{11}$ Pfizer Inc, Montreal, Canada

Background: Tofacitinib is an oral JAK inhibitor for the treatment of psoriatic arthritis (PsA).

Objectives: To assess tofacitinib $5 \mathrm{mg} \mathrm{BID}$ as monotherapy after methotrexate (MTX) withdrawal vs with continued background MTX in patients (pts) with PsA. Methods: OPAL Balance (NCT01976364) was an open-label (OL) long-term extension (LTE) study of tofacitinib in pts with PsA who participated in Phase (P)3 studies (OPAL Broaden, NCT01877668; OPAL Beyond, NCT01882439). Pts who completed $\geq 24$ months' tofacitinib treatment in the LTE (stable $5 \mathrm{mg}$ BID for $\geq 3$ months) and were receiving oral MTX (7.5-20 mg/week; stable for $\geq 4$ weeks) entered the multicentre, 12-month, double-blind, MTX withdrawal substudy. Pts remained on OL tofacitinib $5 \mathrm{mg}$ BID and were randomised 1:1 to receive placebo (tofacitinib monotherapy, ie, blinded MTX withdrawal) or MTX (tofacitinib + MTX same stable doses). Primary endpoints were changes from substudy baseline $(\Delta)$ in PASDAS and HAQ-DI at Month (M)6. Secondary efficacy endpoints were assessed at all time points. Safety was assessed throughout the substudy.

Results: Of 180 pts randomised, 179 were treated (tofacitinib monotherapy $n=90$; tofacitinib + MTX $n=89$ ). Pt characteristics were similar between treatment arms. At M6, least squares mean (LSM) (standard error [SE]) $\triangle$ PASDAS was 0.229 $(0.079)$ for tofacitinib monotherapy and $0.138(0.081)$ for tofacitinib + MTX, and LSM (SE) $\triangle \mathrm{HAQ}$-DI was $0.043(0.027)$ and $0.017(0.028)$, respectively (Figure 1) no clinically meaningful differences were observed. Efficacy and pt-reported outcomes were generally similar between treatment arms at M6 and M12 (data not shown). Rates of pts achieving minimal disease activity, and maintaining an absence of enthesitis and dactylitis, were sustained to M12 in both treatment arms (Figure 2). Adverse event rates (Table) and laboratory parameters were comparable between treatment arms, but liver enzyme elevations were more common with tofacitinib + MTX.

Conclusion: No clinically meaningful differences in efficacy and safety were observed in PsA pts who received OL tofacitinib $5 \mathrm{mg}$ BID as monotherapy after MTX withdrawal vs with continued MTX. Safety was consistent with previous P3 studies. The substudy was an estimation study and not powered for hypothesis testing.

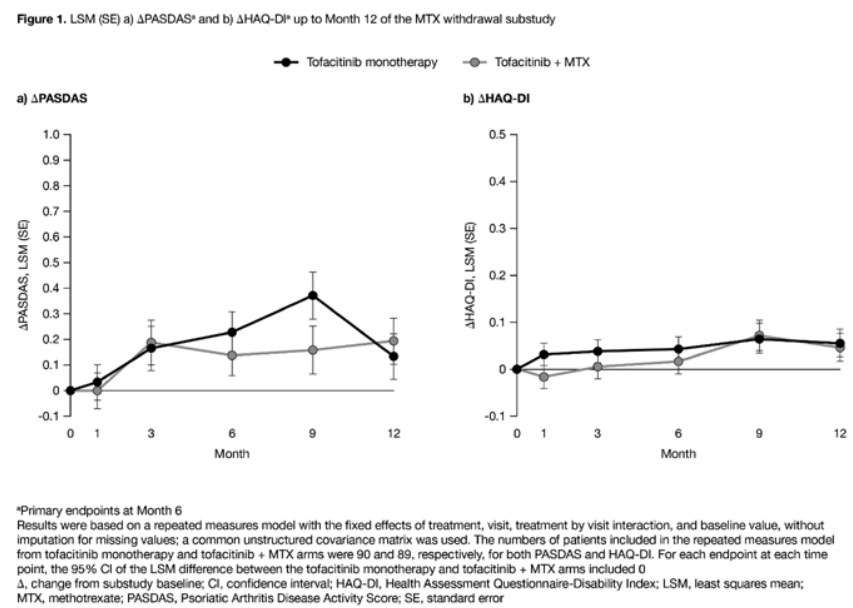

